\title{
CORRESPONDENCE
}

\section{Neonatal emergency transport system during COVID-19 pandemic in the Veneto Region: proposal for standard operating procedures}

Pediatric Research (2021) 89:399-401; https://doi.org/10.1038/s41390020-0937-z

The recent epidemic from a newly isolated coronavirus in Wuhan (China) at the end of 2019 (SARS-CoV-2 or COVID-19) is affecting more than 100 countries, from China to the United States, with Italy having, to date, the highest death toll. ${ }^{1}$

Current evidence suggests that SARS-CoV-2 virus spreads mainly from person-to-person through close contact $(<2 \mathrm{~m})$ and it is transmitted through droplets of the respiratory tract when an infected individual sneezes or coughs. Older people and adults with underlying medical conditions seem at the highest risks, but the impact of the virus in the neonatal population is not yet clear. ${ }^{2}$

Vertical transmission cannot be excluded, although oftentimes neonatal SARS-CoV-2 infection results from postpartum infection or early discharge period if the mother has respiratory symptoms. ${ }^{3}$ There is no evidence for transmission through breastfeeding, thereby this practice is recommended by World Health Organization (WHO) even in mothers with confirmed infection. ${ }^{4}$ However, caution seems still to be required. ${ }^{5}$

There are guidelines on the management of the newborn in a maternity ward and in the Neonatal Intensive Care Unit (NICU), ${ }^{5}$ but no recommendations are available on how to reorganize a Neonatal Emergency Transport Service (NETS).

In the Veneto region, NETS is provided by two Hub centers that cover an area with a population referral base of about five million. Around 400 neonatal transports are carried out per year from 34 spoke hospitals. The staff is composed by a neonatologist, a NICU nurse, a driver, and a Green Cross volunteer.

Here, we report the strategy that was undertaken in the Veneto region, one of the most affected Regions of Italy, to regulate the activity of the NETS during the Covid-19 outbreak.

We aimed to identify safe standardized operational procedures involving the hospitals of the network area, the personnel involved in NETS, and the hub centers. (Fig. 1) Two dedicated ambulances, transport incubators, and emergency bags were exclusively deputed to the transport of at-risk neonates.

\section{MANAGEMENT BEFORE THE DEPARTURE-CONTACT BETWEEN THE SPOKE AND HUB CENTERS}

In addition to routine data included in our transport chart, we have added a space referred to maternal COVID-19 infection. Maternal condition is classified as a "suspect case" or "confirmed case," according to the WHO report. ${ }^{6}$ For every newborn who fulfills the NETS criteria, a specific form for Covid-19 risk infection has to be filled by a parent (Supplementary file).

During the phone call, the healthcare provider confirms the safest way to reach the delivery room or the neonatal ward (e.g., to avoid the transit through the emergency ward, if possible, and the use of "alternative" lifts) at the referring hospital. When the transport is officially confirmed, the transport team gathers at the ambulance with the transport incubator and an emergency bag. The ambulance team loads the incubator onto the ambulance, but it is not allowed to access the space dedicated to the neonate and the transport team. A complete separation among the members of the transport team is maintained to define two operational areas: the space dedicated to the care of the neonate (medical procedural area) and the space dedicated to the ambulance driver (carrier area).

\section{DURING THE NETS}

(a) Stabilization of the newborn: In case of a suspected or confirmed Covid-19-positive newborn, who needs invasive aerosol-generating procedures (i.e., intubation, non-invasive respiratory support, suctioning), the physician and the nurse should wear the individual protective N95 respirator (FFP3 mask), disposable water repellent with long sleeves gown, double gloves, visor/goggles, disposable headgear, and disposable shoes (Fig. 2). Stabilization of the neonate is based on current neonatal resuscitation program and NETS procedures along with appropriate protection of healthcare providers. $^{7}$

In case of a suspected or confirmed Covid-19-positive newborn, who does not require invasive maneuvers, the medical-nursing staff must wear the individual protective N95 mask, disposable gown, gloves, and visor/goggles.

When Covid-19 is not suspected, the management of the newborn follows the standard NETS procedure, but surgical masks and gloves should always be worn by the medical staff and ambulance driver.

Hand washing and use of at least $60 \%$ hydroalcoholic solutions is obligatory during all stages of the transport, as well as the restriction of the minimum number of healthcare providers in the room, avoiding overcrowding. All transport staff have been previously trained for the correct procedure for dressing and undressing. Stabilization procedures are provided based on routine protocol. Closed suctioning systems should be also used. After the patient's stabilization, all the equipment (i.e., masks, laryngoscope, selfinflating bag) should be put in a plastic envelop ready for use during the ambulance transfer.

(b) Management in ambulance: The patient must be positioned in a closed transport incubator, avoiding reopening the portholes, except in case of resuscitation procedures. The doctor and the nurse of the NETS have to wear the N95 respirators, gloves, goggles, and disposable gown.

The number of healthcare providers in the cabin must be minimized (NETS doctor and nurse only), and family members are not allowed.

The loading/unloading of the transport incubator is carried out by the staff of the ambulance with mask and gloves, while making sure the cabin is always kept closed. 


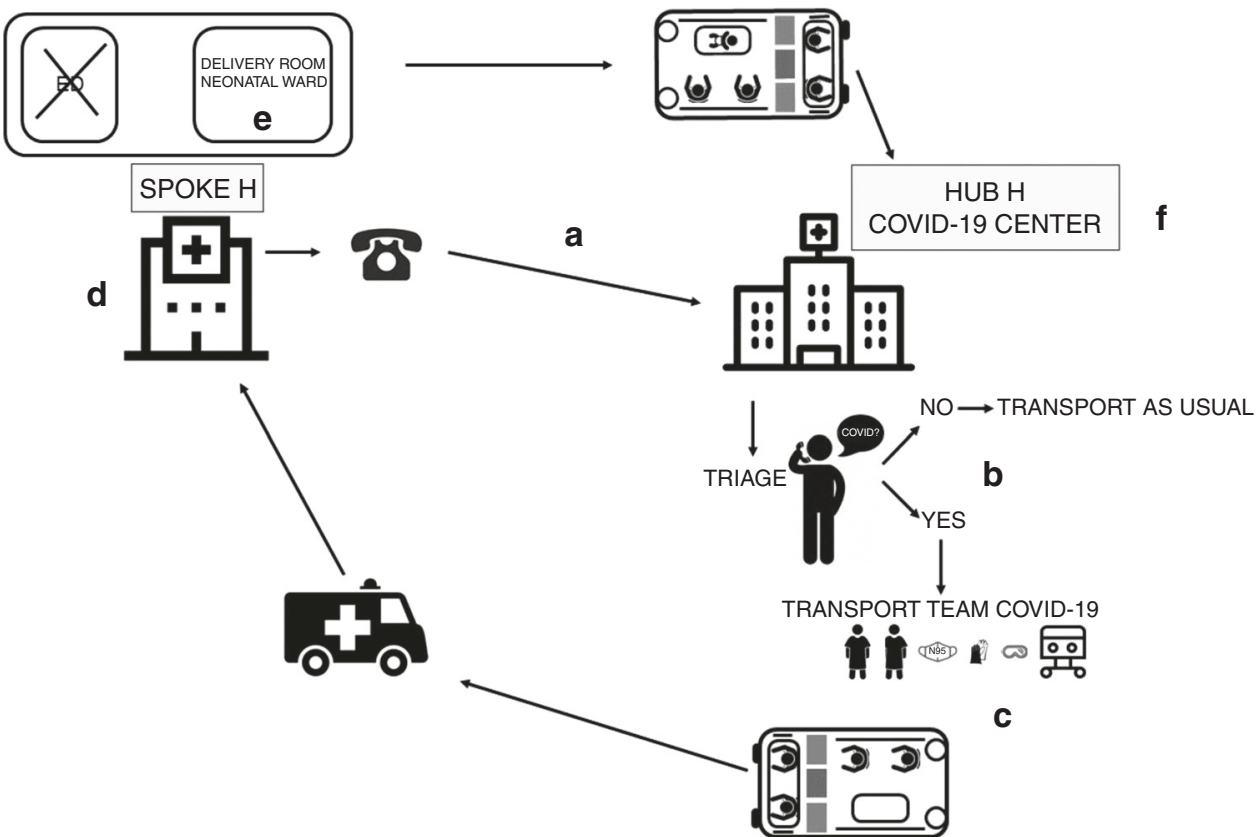

Fig. 1 Neonatal emergency transport organization during COVID-19 pandemic. a Transport request; b collection of (COVID-19) maternal history and patient data; c transport team activation; d arrival at Spoke hospital; e patient stabilization; (f) arrival at COVID-19 Hub hospital.

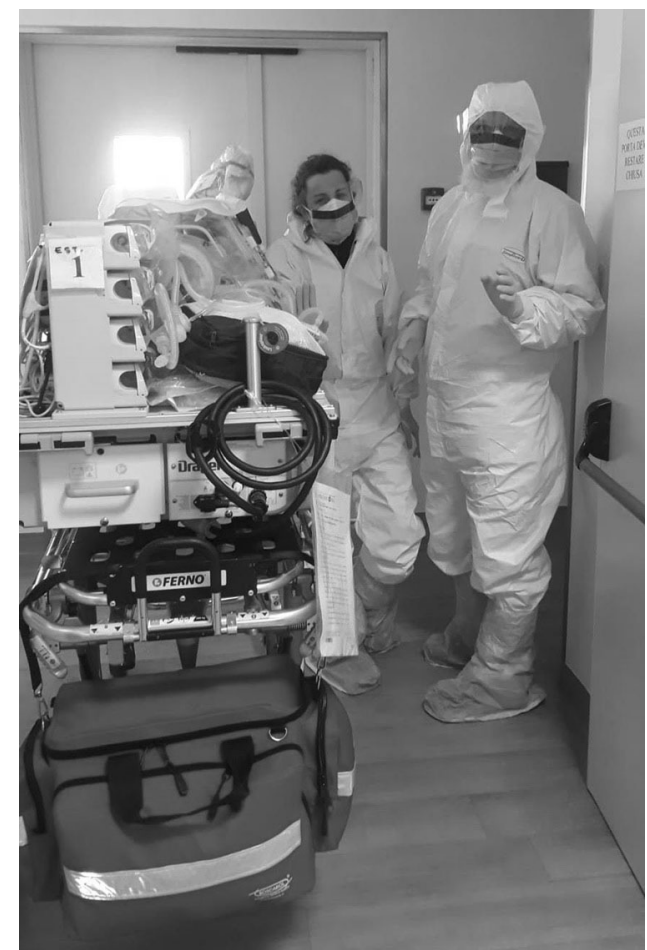

Fig. 2 Nurse (on the left) and doctor (on the right) wearing personal protective equipment during a transport of a COVID-19 suspected newborn. Transport team caring for a neonate with suspected COVID-19 infection.

\section{DESTINATION OF THE PATIENT}

After unloading the transport incubator, the ambulance staff does not escort the NICU's team to the newborn ward, but they wait in the ambulance. The destination of the patient is regulated by a Regional protocol on NETS.

\section{END OF NETS}

Once the patient has been delivered, the nurse is responsible for restoring the material in a new clean emergency bag. The cleaning of the transport incubator and the monitors and ventilator must be done with disinfectant wipes or paper cloth soaked in $70 \%$ ethyl alcohol, diluted $0.5 \%$ chlorine solution, or other approved disinfectant. All the equipment (i.e., masks, laryngoscope, self-inflating bag) should be disposed or sterilized according to standard procedure. All procedures must be performed wearing disposable surgical mask, gloves, and gown.

At the end of the mission, the local emergency medical service is responsible for the sanitization of the vehicle, according to current protocols.

NETS is an essential service, especially during Covid-19 pandemic. It has the role to centralize positive newborns and protect low-risk patients.

Creating a protocol to promptly recognize high-risk cases and define the safest way to reach the newborn, as well as to create a pathway for the best stabilization and ambulance transport, is crucial to minimize the risk of contamination while providing the best possible care for the newborn. Training and simulation are needed to maintain high-quality performance. Further data are urgently needed to produce evidence-based recommendations on NETS during Covid-19 pandemic.

\section{AUTHOR CONTRIBUTIONS}

M.E.C. wrote the initial draft and revised the literature, and approved the final manuscript as submitted. N.D. contributed to write the draft and revised the literature, and approved the final manuscript as submitted. M.A.V. contributed to prepare the protocol, revised the literature, and critically reviewed the manuscript. P.B. contributed to prepare the protocol, provided relevant expertise, and critically reviewed the manuscript. E.B. contributed to coordinate the protocol, provided relevant expertise, and critically reviewed the manuscript. D.T. contributed to the study concept, study design, and writing of the manuscript and critically reviewed the manuscript. All authors approved the final manuscript as submitted and agree to be accountable for all aspects of the work. 


\section{ADDITIONAL INFORMATION}

The online version of this article (https://doi.org/10.1038/s41390-020-0937-z) contains supplementary material, which is available to authorized users.

Competing interests: The authors declare no competing interests.

Publisher's note Springer Nature remains neutral with regard to jurisdictional claims in published maps and institutional affiliations.

Maria Elena Cavicchiolo iD ${ }^{1}$, Nicoletta Doglioni ${ }^{1}$, Mariela Adriana Ventola ${ }^{2}$, Paolo Biban ${ }^{2}$, Eugenio Baraldi ${ }^{1}$ and

Daniele Trevisanuto ${ }^{1}$

${ }^{1}$ Neonatal Intensive Care Unit, Department of Woman's and Child's Health, University Hospital of Padova, Padova, Italy and ${ }^{2}$ Department of Neonatal and Pediatric Critical Care, Verona University Hospital, Verona, Italy Correspondence: Daniele Trevisanuto (daniele.trevisanuto@unipd.it)

\section{REFERENCES}

1. Remuzzi, A. \& Remuzzi, G. COVID-19 and Italy: What next? Lancet 8, 1225-1228 (2020).

2. Bi, Q. et al. Epidemiology and Transmission of COVID-19 in Shenzhen China: Analysis of 391 cases and 1,286 of their close contacts. medRix https://doi.org/ 10.1101/2020.03.03.20028423 (2020).

3. Dong, L. et al. Possible vertical transmission of SARS-CoV-2 from an infected mother to her newborn. JAMA (2020). https://doi.org/10.1001/jama.2020.4621.

4. World Health Organization. Rational use of personal protective equipment for coronavirus disease 2019 (COVID-19): interim guidance. World Health Organization https://apps.who.int/iris/handle/10665/331215 (2020).

5. Wang, L. et al. Chinese expert consensus on the perinatal and neonatal management for the prevention and control of the 2019 novel coronavirus infection (First edition). Ann. Transl. Med. 8, 47 (2020).

6. World Health Organization. Coronavirus disease (COVID-2019) situation reports World Health Organization https://www.who.int/emergencies/diseases/novelcoronavirus-2019/situation-reports/ (2020).

7. Chandrasekharan, P. et al. Neonatal resuscitation and post-resuscitation care of infants born to mothers with suspected or confirmed SARS-CoV-2 infection. Am. J. Perinatol. (2020). https://doi.org/10.1055/s-0040-1709688. 